\title{
Complete response with early introduction of cabazitaxel in a patient with multiple lung metastases of castration-resistant prostate cancer following the early detection of metastases using liquid biopsy: a case report
}

Takeo Kosaka* (iD, Hiroshi Hongo and Mototsugu Oya

\begin{abstract}
Background: Cabazitaxel (CBZ) chemotherapy for metastatic castration-resistant prostate cancer (mCRPC) is believed to be palliative because the radiological response rate is low and a durable response is rare. Here, we describe a rare case of a patient with MCRPC who was treated with CBZ chemotherapy and showed a durable radiological response and a complete biochemical response.

Case presentation: A 43-year-old man with prostate cancer and metastasis of the pubic bone underwent neoadjuvant androgen deprivation and docetaxel therapy, followed by laparoscopic prostatectomy, extended lymphadenectomy, and metastatectomy in 2014. Pathological examination revealed residual adenocarcinoma in the prostate and pubic bone (pathological T stage 3b, positive surgical margin). Following the operation, he received adjuvant radiation therapy (66 Gy) to the pelvic floor. His serum prostate-specific antigen (PSA) level decreased to $<0.01 \mathrm{ng} / \mathrm{mL}$ but gradually increased following docetaxel chemotherapy. Imaging findings indicated five tiny nodules in the bilateral lungs. Biopsy specimens are difficult to obtain and might not reflect the precise extent of the disease owing to heterogeneity in patients with CRPC. Thus, we performed liquid biopsy to isolate circulating tumor cells (CTCs), and overall 156 CTCs were detected per $7.5 \mathrm{~mL}$. Almost all CTCs were androgen receptornegative in the nucleus. We diagnosed the five nodules as lung metastases from docetaxel-resistant CRPC with few AR-signaling-dependent cancer cells. The patient was initiated on CBZ chemotherapy $\left(25 \mathrm{mg} / \mathrm{m}^{2}\right)$ according to the standard protocol in August 2016, instead of using a second-generation AR-targeting agent. After 2 cycles of CBZ chemotherapy, PSA level decreased to $<0.01 \mathrm{ng} / \mathrm{mL}$ and the lung metastases completely disappeared, with a reduced CTC count of $<5$. To date, the patient has been receiving intermittent CBZ chemotherapy.
\end{abstract}

Conclusions: We presented a rare case of a patient with $\mathrm{MCRPC}$ who was successfully treated with early CBZ chemotherapy. The early detection of metastasis using liquid biopsy enabled the introduction of early CBZ chemotherapy for docetaxel-resistant MCRPC.

Keywords: Cabazitaxel, Castration-resistant prostate cancer, Circulating tumor cells

\footnotetext{
* Correspondence: takemduro@gmail.com; takemduro@keio.jp Department of Urology, Keio University School of Medicine, 35 Shinanomachi, Shinjuku-ku, Tokyo 160-8582, Japan
}

(c) The Author(s). 2019 Open Access This article is distributed under the terms of the Creative Commons Attribution 4.0 International License (http://creativecommons.org/licenses/by/4.0/), which permits unrestricted use, distribution, and reproduction in any medium, provided you give appropriate credit to the original author(s) and the source, provide a link to the Creative Commons license, and indicate if changes were made. The Creative Commons Public Domain Dedication waiver (http://creativecommons.org/publicdomain/zero/1.0/) applies to the data made available in this article, unless otherwise stated. 


\section{Background}

Cabazitaxel (CBZ) is a next-generation taxane that is indicated for the treatment of patients with metastatic castration-resistant prostate cancer (mCRPC) who were previously treated using a docetaxel-containing regimen [1]. However, CBZ chemotherapy for patients with $\mathrm{mCRPC}$ is believed to be palliative because the radiological response rate is low and a durable response is rare. Here, we describe a rare case of a patient with mCRPC who was treated with CBZ chemotherapy and demonstrated a durable radiological response and a complete biochemical response.

\section{Case presentation}

A 43-year-old man was diagnosed with metastatic prostate cancer (Gleason score $4+4$ ) in November 2013. Laboratory data showed that the prostate-specific antigen (PSA) level was $18.6 \mathrm{ng} / \mathrm{mL}$, and digital rectal examination indicated a stony hard mass in the prostate that was suspected to be local advanced prostate cancer. Magnetic resonance imaging revealed a prostate tumor invading the seminal vesicle and a metastasis of the pubic bone (Fig. 1a). Based on these results, the patient underwent neoadjuvant androgen deprivation and docetaxel therapy, followed by laparoscopic prostatectomy, extended lymphadenenolectomy, and metastatectomy of the pubic bone in March 2014. Pathological examination revealed residual adenocarcinoma in the prostate and pubic bone (pathological $\mathrm{T}$ stage $3 \mathrm{~b}$, positive surgical margin). After the operation, he received adjuvant radiation therapy (66 Gy) to the pelvic floor. His serum PSA level decreased to $<0.01 \mathrm{ng} / \mathrm{mL}$ but gradually increased to $0.14 \mathrm{ng} / \mathrm{mL}$. He was then re-initiated on docetaxel in
December 2015, although computed tomography (CT) and bone scan did not show obvious metastatic lesions. His PSA level decreased to $<0.01 \mathrm{ng} / \mathrm{mL}$ in April 2016 after 7 cycles of docetaxel chemotherapy but slightly increased to $0.17 \mathrm{ng} / \mathrm{mL}$ in July 2016. Positron emission tomography-CT indicated five tiny nodules in the bilateral lungs (Fig. 1b). Biopsy specimens are difficult to obtain and might not reflect the precise extent of the disease owing to heterogeneity in patients with CRPC. Therefore, we performed liquid biopsy to isolate circulating tumor cells (CTCs) using the ClearCell FX System, which is an automated CTC enrichment system that is powered by a microfluidics biochip [2]. To count the CTCs isolated using this system, we performed immunostaining using the following antibodies: mouse anti-pan human keratin (C11) monoclonal antibody (mAb) (keratin 4, 5, 6, 8, 10, 13, and 18; Cell Signaling, Danvers, MA, USA), mouse anti-human cytokeratin mAb (CK3-6H5, Miltenyi Biotec GmbH, Bergisch Gladbach, Germany), mouse anti-human EpCAM (VU1D9) $\mathrm{mAb}$ (Cell Signaling), goat N-terminal androgen receptor (AR; N-10) polyclonal antibody (Santa Cruz Biotechnology, Santa Cruz, CA, USA), and rabbit CD45 (D9M81) $\mathrm{mAb}$ (Cell Signaling). Additionally, we performed nuclear staining using 4',6-diamidino-2-phenylindole [2]. Overall, 156 CTCs were detected per $7.5 \mathrm{~mL}$, and almost all CTCs were AR negative in the nucleus (Fig. 2). Therefore, we diagnosed the five tiny nodules as lung metastases from docetaxel-resistant CRPC with few AR-signalingdependent cancer cells.

The patient was initiated on CBZ $\left(25 \mathrm{mg} / \mathrm{m}^{2}\right)$ according to the standard protocol in August 2016, instead of a second-generation AR-targeting agent (enzalutamide or
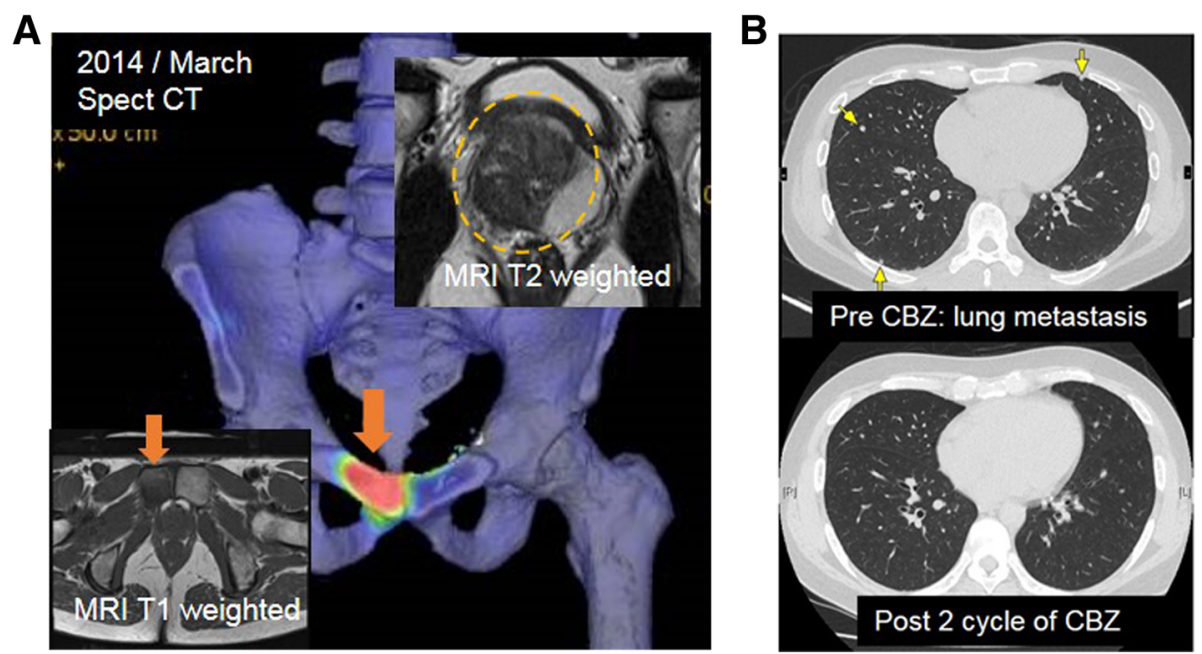

Fig. 1 a Magnetic resonance imaging and bone single-photon emission computerized tomography show a prostate tumor invading the seminal vesicle and bone metastasis in the pubic bone. $\mathbf{b}$ Positron emission tomography-computed tomography shows tiny nodules in the bilateral lungs. Following 2 cycles of cabazitaxel chemotherapy, the lung metastases completely disappeared 


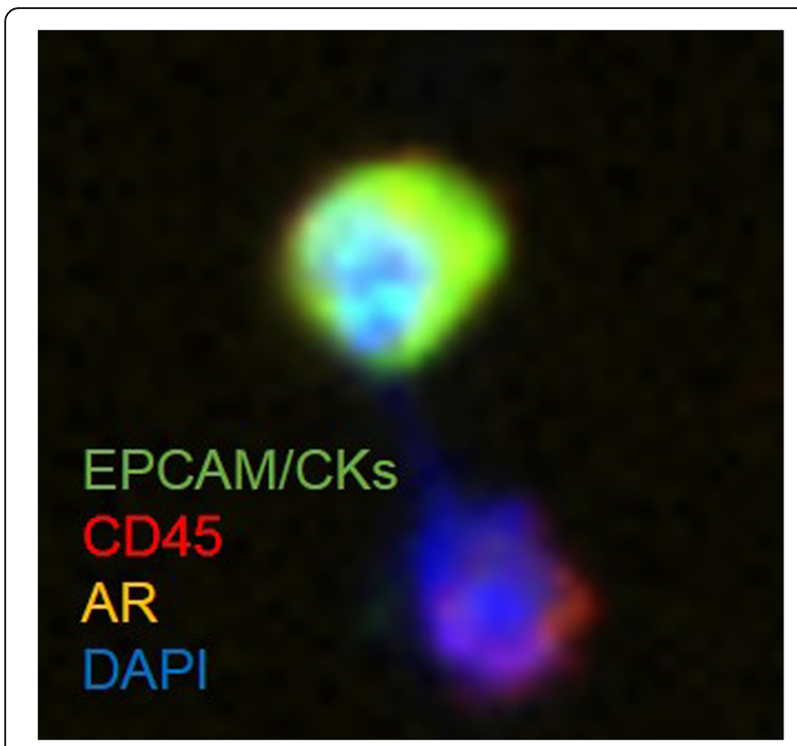

Fig. 2 An automated circulating tumor cell (CTC) enrichment system powered by a microfluidics biochip detects CTCs that are androgen receptor-negative in the nucleus

abiraterone) [3]. Following 2 cycles of CBZ chemotherapy, the PSA level decreased to $<0.01 \mathrm{ng} / \mathrm{mL}$ and the lung metastases completely disappeared, with a reduced CTC count of $<5$. To date, the patient has been receiving intermittent CBZ chemotherapy.

\section{Discussion}

We experienced a patient with $\mathrm{mCRPC}$ who was successfully treated with CBZ chemotherapy. The patient exhibited multiple lung metastases of CRPC, and a complete response was noted with the early introduction of CBZ.

Unmet needs in patient management include the ability to monitor treatment effects and the early detection of metastasis. A common shortcoming of a CTC enrichment system is its reliance on the positive selection of CTCs with antibodies against EpCAM, suggesting the limited ability to identify CTCs with reduced EpCAM expression because of epithelial-mesenchymal transition (EMT) [4-6]. In fact, in our patient, antibodies targeting EpCAM and CD45 were only detected in $31 \%$ of CTCs, suggesting the limited ability to detect CTCs according to EpCAM. Microfluidics involves several separation methods that facilitate the manipulation of extremely small volumes of biological fluids, thereby enabling the detection of de-differentiated CRPC with EMT.

\section{Conclusion}

We present a rare case of a patient with $\mathrm{mCRPC}$ who was successfully treated with early CBZ chemotherapy. The early detection of metastasis using liquid biopsy enabled the introduction of early CBZ chemotherapy for docetaxel-resistant mCRPC.

\section{Abbreviations}

CBZ: Cabazitaxel; CTCs: Circulating tumor cells; EMT: Epithelial-mesenchymal transition; $\mathrm{MCRPC}$ : metastatic castration-resistant prostate cancer

\section{Acknowledgements}

Eriko Suzuki provided technical assistance.

\section{Authors' contributions}

$\mathrm{TK}, \mathrm{HH}$, and $\mathrm{MO}$ made substantial contributions to conception and design. $\mathrm{TK}$ and $\mathrm{HH}$ made substantial contributions to the laboratory analysis and interpretation of data; TK and MO made substantial contributions to the acquisition of clinical data and their interpretation. TK and $\mathrm{HH}$ have been involved in drafting the manuscript. MO has been involved in critically revising the manuscript for important intellectual content. $\mathrm{TK}, \mathrm{HH}$, and $\mathrm{MO}$ gave their final approval of the version to be published.

\section{Funding}

This study was supported in part by Grants-in-Aid for Scientific Research (\#17 K11158 to T. Kosaka) from the Ministry of Education, Culture, Sports, Science, and Technology of Japan. This funding supported the collection of CTC. The study was supported in part by research Grant to T. Kosaka from the Takeda Science Foundation, Japan. The funding supported the analysis of CTC.

Availability of data and materials Not applicable.

\section{Ethics approval and consent to participate}

Our study was designed as a retrospective analysis and approval was obtained from the Keio University School of Medicine an Ethical Committee (\#20140159 and \#20150242).

\section{Consent for publication}

Written informed consent was obtained from the patient for publication of this case report.

\section{Competing interests}

The authors declare that they have no competing interests.

Received: 5 September 2018 Accepted: 31 May 2019

Published online: 11 June 2019

\section{References}

1. de Bono JS, Oudard S, Ozguroglu M, Hansen S, Machiels JP, Kocak I, et al. Prednisone plus cabazitaxel or mitoxantrone for metastatic castrationresistant prostate cancer progressing after docetaxel treatment: a randomised open-label trial. Lancet. 2010;376(9747):1147-54.

2. Hou HW, Warkiani ME, Khoo BL, Li ZR, Soo RA, Tan DS, et al. Isolation and retrieval of circulating tumor cells using centrifugal forces. Sci Rep. 2013;3:1259.

3. Kosaka T, Shinojima T, Morita S, Oya M. Prognostic significance of grade $3 / 4$ neutropenia in Japanese prostate cancer patients treated with cabazitaxel. Cancer Sci. 2018;109(5):1570-5.

4. Armstrong AJ, Marengo MS, Oltean S, Kemeny G, Bitting RL, Turnbull JD, et al. Circulating tumor cells from patients with advanced prostate and breast cancer display both epithelial and mesenchymal markers. Mol Cancer Res. 2011;9(8):997-1007.

5. Beltran $H$, Jendrisak A, Landers M, Mosquera JM, Kossai M, Louw J, et al. The initial detection and partial characterization of circulating tumor cells in neuroendocrine prostate Cancer. Clin Cancer Res. 2016;22(6):1510-9.

6. Miyamoto DT, Sequist LV, Lee RJ. Circulating tumour cells-monitoring treatment response in prostate cancer. Nat Rev Clin Oncol. 2014;11(7):401-12.

\section{Publisher's Note}

Springer Nature remains neutral with regard to jurisdictional claims in published maps and institutional affiliations. 